\title{
Endo-COVID-19: la risposta delle Scuole di Endocrinologia e Malattie del Metabolismo Italiane alla pandemia da SARS-COV2
}

\author{
Andrea M. Isidori ${ }^{1}$ - Emmanuele A. Jannini ${ }^{2}$. Andrea Lenzi ${ }^{1}$ - Ezio Ghigo ${ }^{3}$
}

Pubblicato online: 22 maggio 2020

(c) Springer Nature Switzerland AG 2020

L'infezione da virus SARS-CoV-2, un coronavirus mutato che si è diffuso verosimilmente dall'oriente a partire dagli ultimi mesi dello scorso anno e dotato di spiccato tropismo respiratorio, in alcuni soggetti determina un' aberrante risposta infiammatoria con evidente interessamento internistico per coinvolgimento polmonare, cardiovascolare, coagulativo e nervoso. Proprio per l'intrinseca natura internistica, molti reparti di assistenza e assistenza-formazione specialistica di endocrinologia sono stati chiamati a collaborare agli sforzi di riorganizzazione dei nosocomi destinati a fronteggiare l'epidemia.

Tra l' 8 e il 14 aprile è stato chiesto ai Direttori delle Scuole di Specializzazione di compilare un questionario relativo al contributo che ciascuna sede ha fornito in tal senso. L'indagine è stata condotta solo su questo specifico campione per l'omogeneità e facoltà di verifica in tempi rapidi ma riteniamo che sia espressione del contributo che tutta l'endocrinologia nazionale sta dando all'emergenza.

Attraverso una survey online è stato chiesto di fornire il numero di unità di personale medico, strutturato e in formazione, personale infermieristico e tecnico, degenze ordinarie e day hospital (DH), che sono state messe a disposizione per la riorganizzazione della rete assistenziale nel perio-

Per il Collegio dei Professori Ordinari di Endocrinologia, Metabolismo e Andrologia (Collegium P.O.E.M.A.) e per i Direttori delle Scuole di Specializzazione in Endocrinologia e Malattie del Metabolismo.

$凶$ E. Ghigo

ezio.ghigo@unito.it

1 Dipartimento di Medicina Sperimentale, Sapienza Università di Roma, Roma, Italia

2 Dipartimento di Medicina dei Sistemi, Università di Roma Tor Vergata, Roma, Italia

3 Dipartimento di Scienze Mediche, Università di Torino, Torino, Italia do coincidente con la prima fase del lock-down (7 marzo-7 aprile).

L'adesione è stata completa da parte di tutte le Scuole con un tasso di compilazione del $100 \%$ delle domande, per un totale di 34 Scuole partecipanti, espressione del coinvolgimento di 35 Università $^{1}$ e di 45 Aziende Sanitarie. ${ }^{2}$

${ }^{1}$ Strutture universitarie e le relative strutture sanitarie su cui insistono le Scuole oggetto della survey Università: Università di Torino, Università Piemonte Orientale, Università dell'Insubria, Università di Milano, Università di Milano Bicocca, Università di Milano Vita-Salute San Raffaele, Humanitas University Milano, Università di Pavia, Università di Brescia, Università di Verona, Università di Padova, Università degli Studi di Genova, Università di Parma, Università di Ferrara, Università di Modena e Reggio Emilia, Università di Bologna Alma Mater Studiorum, Università di Pisa, Università di Firenze, Università di Siena, Università Politecnica delle Marche, Università di Perugia, Sapienza Università di Roma, Università di Roma Tor Vergata, Università Cattolica del Sacro Cuore di Roma, Campus Bio-Medico di Roma, Università dell'Aquila, Università di Chieti-Pescara G. d'Annunzio, Università di Napoli Federico II, Università della Campania Luigi Vanvitelli, Università degli Studi di Bari Aldo Moro, Università di Catanzaro Magna Graecia, Università di Messina, Università di Catania, Università di Palermo, Università di Cagliari.

${ }^{2}$ Strutture sanitarie: Struttura Complessa (SC) Endocrinologia, Diabetologia e Metabolismo e SC Endocrinologia Oncologica, Azienda Ospedaliero-Universitaria (AOU) "Città della Salute e della Scienza" (Torino), AOU "Maggiore della Carità" di Novara (Novara), Azienda Socio Sanitaria Territoriale (ASST) dei Sette Laghi di Varese (Varese), Policlinico di Milano (Milano), Istituto Auxologico Italiano (Milano), Ospedale Fatebenefratelli (Milano), Policlinico di Monza (Monza), Ospedale Papa Giovanni XXIII di Bergamo (Bergamo), Istituti di Ricovero e Cura a Carattere Scientifico (IRCCS) Ospedale San Raffaele (Milano), IRCCS Istituto Clinico HUMANITAS (Milano), Unità Operativa Complessa (UOC) di Medicina Interna ed Endocrinologia, Istituti Clinici Scientifici (ICS) Maugeri IRCCS di Pavia (Pavia), ASST Spedali Civili Brescia (Brescia), UOC Endocrinologia, Diabetologia e Malattie del Metabolismo, AOU integrata Verona (Verona), AOU di Padova (Padova), IRCCS Policlinico San Martino (Genova), AOU di Parma (Parma), AOU di Ferrara (Ferrara), UOC di Endocrinologia, AOU di Modena (Modena), AOU Policlinico S.Orsola-Malpighi 


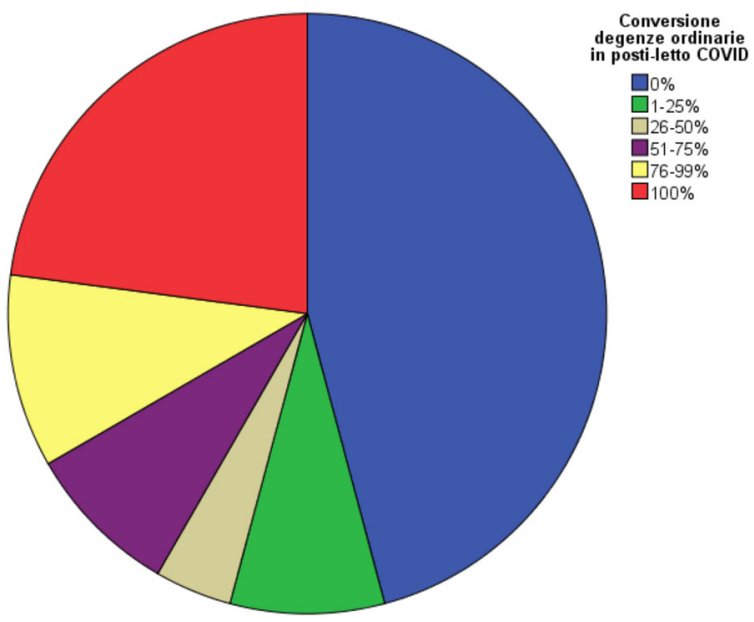

Fig. 1 Conversione degenze ordinarie

L'analisi è di seguito rappresentata su scala nazionale e per aree macroregionali, per il diverso impatto epidemiologico nelle aree coinvolte. Nello specifico sono stati utilizzati tre raggruppamenti delle regioni che esprimono Scuole di Specializzazione in endocrinologia: Nord (Piemonte, Liguria, Lombardia, Veneto, con l'Emilia Romagna), Centro (Toscana, Lazio, Abruzzo, Marche, Umbria), Sud e isole (Campania, Puglia, Calabria, Sicilia, Sardegna) (Tabella 1).

Nel 22,9\% dei casi, l'intero reparto di endocrinologia è stato convertito in posti letti per l'emergenza COVID (36,8\% al Nord), mentre un contributo pari ad almeno il 50\% dei posti letto ordinari è stato dato nel $41,7 \%$ su scala nazionale, nel $68,4 \%$ al Nord, e nel $25,1 \%$ e $23,1 \%$, rispettivamente, al Centro e al Sud. A livello nazionale, il 45,8\% dei reparti ha mantenuto invariata la propria attività di ricovero, ma solo il 26,3\% al Nord (Fig. 1).

Per quanto riguarda la ricollocazione del personale specialistico (endocrinologi strutturati nel Sistema Sanitario Nazionale), nel 15,8\% dei casi dei reparti del Nord Italia, l'intero gruppo della struttura è stato ricollocato in attività

di Bologna (Bologna), AOU Pisana (Pisa), AOU Careggi di Firenze (Firenze), AOU Senese (Siena), Ospedali Riuniti Torrette di Ancona - Umberto I (Ancona), UOC di Endocrinologia e Malattie Metaboliche, Azienda Ospedaliera di Perugia (Perugia), AOU Policlinico Umberto I di Roma (Roma), AOU Sant'Andrea di Roma (Roma), Ospedale S. Maria Goretti di Latina (Latina), Fondazione Policlinico Tor Vergata Di Roma (Roma), Fondazione Policlinico Agostino Gemelli di Roma (Roma), Ospedale Ramazzini di Carpi (Modena), Ospedale Regina Apostolorum, Albano Laziale (Roma), ASL Frosinone (Frosinone), ASL Roma 2 (Roma), Ospedale Regionale S. Salvatore (L'Aquila), Azienda USL Pescara (Pescara), AOU Policlinico Federico II (Napoli), AOU dell'Università della Campania Luigi Vanvitelli (Napoli), AOU Policlinico Consorziale di Bari (Bari), Azienda Ospedaliera “Ospedali Riuniti” di Foggia (Foggia), AOU Materdomini, Policlinico Universitario (Germaneto), UOC di Endocrinologia, AOU Policlinico G. Martino di Messina (Messina), ARNAS Garibaldi, Catania (Catania), AOU Policlinico di Catania (Catania), AOU Policlinico, Ospedale Paolo Giaccone (Palermo), Ospedale Cervello (Palermo), Ospedale di Partinico (Palermo), AOU "Duilio Casula" di Cagliari (Cagliari).

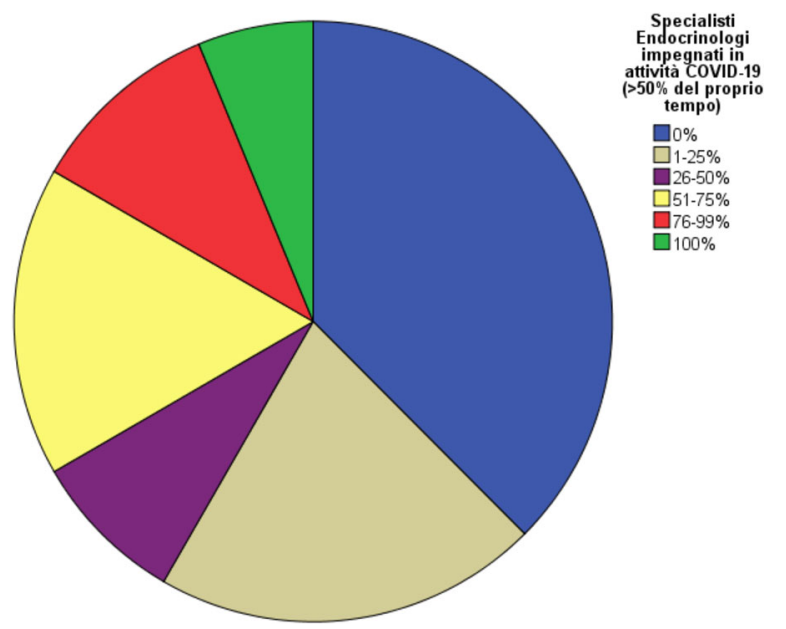

Fig. 2 Specialisti endocrinologi impegnati in attività COVID-19

COVID-19, mentre su scala nazionale questo numero scende al 6,3\% (Fig. 2). Tuttavia, in ben il 63,2\% delle strutture del Nord almeno metà del personale specialistico è stato utilizzato in attività COVID e, comunque, in oltre un terzo delle strutture $(33,4 \%)$ su scala nazionale. In termini di unità, significa che su scala nazionale ben 168 endocrinologi strutturati hanno dedicato la maggior parte della loro assistenza alla gestione sanitaria COVID (di questi 141 al Nord, 31 al Centro e 9 al Sud). Ovviamente, questi numeri si riferiscono ai medici che sono stati direttamente impiegati nella gestione delle degenze COVID, mentre i rimanenti hanno comunque coperto i servizi rimasti operativi o coperto gli altri servizi lasciati scoperti dalla riorganizzazione della rete ospedaliera.

Anche il personale infermieristico è stato ricollocato per coprire l'emergenza. Il trasferimento di tutte le unità è stato necessario nel $12,5 \%$ del territorio nazionale $(21,1 \%$ al Nord), mentre il trasferimento di almeno la metà del personale infermieristico è avvenuto nel 35,4\% (Nord: 52,7\%). In termini di unità significa che su scala nazionale ben 192 infermieri hanno dedicato la maggior parte della loro assistenza alla gestione sanitaria COVID (di questi 130 al Nord, 44 al Centro e 28 al Sud).

La riorganizzazione ha comportato una riduzione o sospensione dell'attività di ricovero in $\mathrm{DH}$ per l' $89,6 \%$ delle strutture (Fig. 3) ma, nonostante la sospensione di tali ricoveri, le attività ambulatoriali con la copertura delle prestazioni urgenti e non differibili è stata garantita nella quasi totalità dei centri (Fig. 4).

Per quanto riguarda gli specializzandi, anche su questo fronte si registra un notevole contributo alla crisi sanitaria. Un terzo delle Scuole su scala nazionale ha messo a disposizione oltre la metà dei propri specializzandi $(31,2 \%)$, e un aggiuntivo $16,7 \%$ ha messo a disposizione una quota inferiore. Tradotto in numero, questo corrisponde a un "piccolo esercito" di almeno 190 giovani endocrinologi in formazio- 
Tabella 1 Il contributo delle Scuole di Endocrinologia e Malattie del Metabolismo all'emergenza Covid-19

\begin{tabular}{|c|c|c|c|c|}
\hline & Totale & Nord & Centro & Sud/Isole \\
\hline Scuole (n) & 34 & 16 & 11 & 7 \\
\hline - Poli Unici & 26 & 14 & 8 & 4 \\
\hline - Poli Multipli & 8 & 2 & 3 & 3 \\
\hline Specialisti endocrinologi afferenti alla struttura sanitaria (n) & 502 & 223 & 163 & 116 \\
\hline Specializzandi afferenti alla sede (n) & 403 & 166 & 145 & 92 \\
\hline Infermieri/tecnici afferenti alla struttura sanitaria (n) & 543 & 247 & 174 & 122 \\
\hline \multicolumn{5}{|l|}{ Degenze ordinarie convertite in posti COVID (\%) } \\
\hline$-0 \%$ & 45,8 & 26,3 & 62,4 & 53,8 \\
\hline$-1-25 \%$ & 8,3 & 0 & 12,5 & 15,4 \\
\hline$-26-50 \%$ & 4,2 & 5,3 & 0 & 7,7 \\
\hline$-51-75 \%$ & 8,3 & 15,8 & 6,3 & 0 \\
\hline$-75-99 \%$ & 10,5 & 15,8 & 12,5 & 0 \\
\hline$-100 \%$ & 22,9 & 36,8 & 6,3 & 23,1 \\
\hline \multicolumn{5}{|c|}{ Specialisti impegnati (per oltre il 50\% del proprio tempo) in attività dei reparti/servizi COVID? (\%) } \\
\hline$-0 \%$ & 37,5 & 10,5 & 43,7 & 69,2 \\
\hline$-1-25 \%$ & 20,8 & 15,8 & 25 & 23,1 \\
\hline$-26-50 \%$ & 8,3 & 10,5 & 12,5 & 0 \\
\hline$-51-75 \%$ & 16,7 & 26,3 & 18,8 & 0 \\
\hline$-76-99 \%$ & 10,4 & 21,1 & 0 & 7,7 \\
\hline$-100 \%$ & 6,3 & 15,8 & 0 & 0 \\
\hline \multicolumn{5}{|c|}{ Infermieri impegnati (per oltre il $50 \%$ del proprio tempo) in attività dei reparti/servizi COVID? $(\%)$} \\
\hline$-0 \%$ & 35,4 & 21,1 & 50 & 38,5 \\
\hline$-1-25 \%$ & 25 & 15,8 & 25 & 38,5 \\
\hline$-26-50 \%$ & 4,2 & 10,4 & 0 & 0 \\
\hline$-51-75 \%$ & 10,4 & 15,8 & 12,5 & 0 \\
\hline$-76-99 \%$ & 12,5 & 15,8 & 12,5 & 7,7 \\
\hline$-100 \%$ & 12,5 & 21,1 & 0 & 15,3 \\
\hline \multicolumn{5}{|l|}{ L'attività di ricovero in $\mathrm{DH}(\%)$} \\
\hline - È continuata regolarmente & 4,2 & 5,3 & 6,3 & 0 \\
\hline - È stata ridotta & 43,8 & 36,8 & 43,8 & 53,8 \\
\hline - È stata sospesa & 45,8 & 52,6 & 37,4 & 46,2 \\
\hline- Altro & 6,2 & 5,3 & 12,5 & 0 \\
\hline \multicolumn{5}{|l|}{ La continuità dei servizi ambulatoriali di endocrinologia $(\%)$} \\
\hline - È garantita solo per visite urgenti e non differibili & 81,3 & 89,4 & 75 & 76,9 \\
\hline - È garantita solo per visite urgenti e non differibili + telemedicina & 10,4 & 5,3 & 6,3 & 23,1 \\
\hline - È mantenuta per tutte le tipologie di visite ma ridotta a meno del $25 \%$ & 6,3 & 5,3 & 12,4 & 0 \\
\hline - È stata sospesa & 2 & 0 & 6,3 & 0 \\
\hline - Altro & 0 & 0 & 0 & 0 \\
\hline \multicolumn{5}{|c|}{ Specializzandi impegnati (per oltre il 50\% del proprio tempo) in attività dei reparti/servizi COVID? (\%) } \\
\hline$-0 \%$ & 52,1 & 5,3 & 68,6 & 100 \\
\hline$-1-25 \%$ & 10,4 & 15,3 & 12,5 & 0 \\
\hline$-26-50 \%$ & 6,3 & 10,5 & 6,3 & 0 \\
\hline$-51-75 \%$ & 8,3 & 15,8 & 6,3 & 0 \\
\hline$-76-99 \%$ & 8,3 & 21,1 & 0 & 0 \\
\hline$-100 \%$ & 14,6 & 32 & 6,3 & 0 \\
\hline
\end{tabular}


Tabella 1 (Continued)

\begin{tabular}{|c|c|c|c|c|}
\hline & Totale & Nord & Centro & Sud/Isole \\
\hline \multicolumn{5}{|c|}{ Il trasferimento del personale medico è avvenuto su base } \\
\hline - Obbligatoria & 27,1 & 21,1 & 31,0 & 30,8 \\
\hline - Volontaria & 41,7 & 57,9 & 43,8 & 15,4 \\
\hline - Non c'è stato trasferimento & 12,5 & 0 & 6,3 & 38,4 \\
\hline - Su proposta della direzione & 4,2 & 10,5 & 0 & 0 \\
\hline - Altro & 14,5 & 10,5 & 18,9 & 15,4 \\
\hline \multicolumn{5}{|c|}{ Il trasferimento del personale infermieristico è avvenuto su base } \\
\hline - Obbligatoria & 50 & 52,6 & 50 & 46,2 \\
\hline - Volontaria & 18,8 & 15,8 & 18,8 & 23 \\
\hline - Non c'è stato trasferimento & 18,8 & 15,8 & 25 & 15,4 \\
\hline - Su proposta della direzione & 4,2 & 10,5 & 0 & 0 \\
\hline - Altro & 8,2 & 5,3 & 6,2 & 15,4 \\
\hline
\end{tabular}

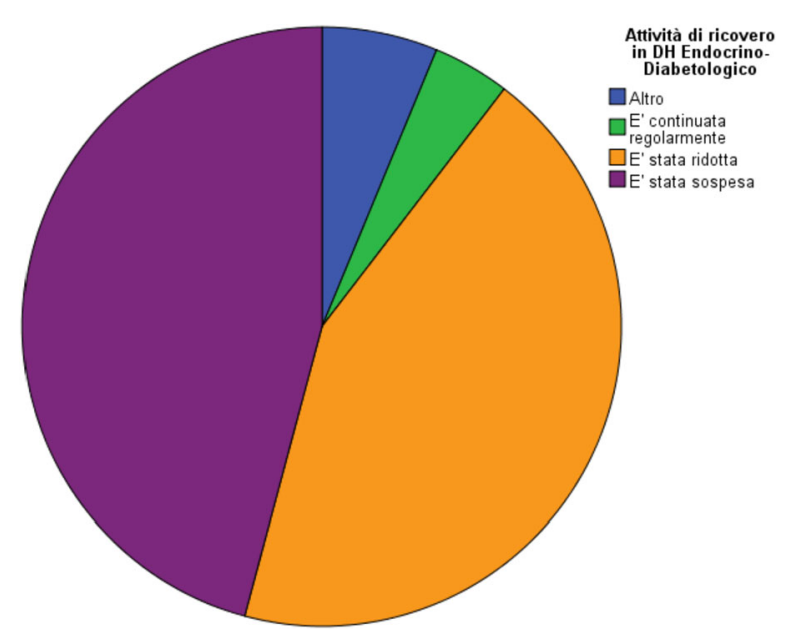

Fig. 3 Gestione ricoveri in DH endocrino-metabolico

ne che si sono, o sono stati, parzialmente o completamente messi a disposizione dell'emergenza sanitaria (Fig. 5).

Il coinvolgimento degli specializzandi, tuttavia, è molto più alto nel Nord $(68,4 \%$ con più della metà degli specializzandi afferenti e un addizionale $26,3 \%$ in quote minori), presente ma significativamente minore al Centro (rispettivamente 12,6 e 18,8\%), mentre al Sud e nelle isole non risulta che gli specializzandi di endocrinologia siano stati direttamente coinvolti in attività connesse con l'emergenza COVID-19. Ovviamente, questo dato deve essere interpretato alla luce del diverso impatto che la pandemia ha avuto nel nord, rispetto al centro e sud Italia (e probabilmente anche alla disponibilità dei dispositivi di protezione individuale, DPI).

Un aspetto che questa survey ha messo in luce riguarda l'adesione alla gestione dell'emergenza, avvenuta su base volontaria o su proposta della direzione nel $45,9 \%$ dei casi (obbligatoria nel $27,1 \%$ ). In molti casi, la motivazione dei

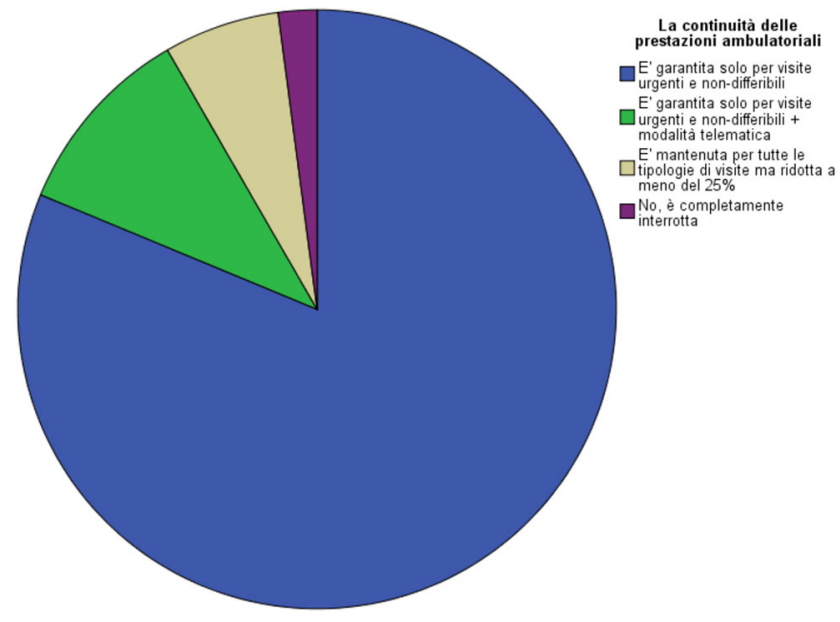

Fig. 4 Gestione pazienti ambulatoriali

medici e degli specializzandi a voler dare un contributo è stata descritta e riportata con orgoglio dai Direttori di Scuola che hanno compilato la survey.

In sintesi, questa Survey dimostra la capacità dell'Endocrinologia, in tutte le sue declinazioni, endocrinologica generale, diabetologica, metabolica e andrologica, di fornire un valido supporto alla tenuta del nostro sistema sanitario, fornendo competenza, personale e solidarietà. La nostra risposta all'emergenza non è stata soltanto fornire una guida alla migliore gestione del paziente endocrinometabolico con COVID-19 [1-5], ma anche una massiccia mobilitazione di risorse umane.

Riteniamo che questo documento debba essere motivo di orgoglio per tutte le Scuole di Endocrinologia e Malattie del Metabolismo italiane, per i Direttori, i Coordinatori e per tutti i docenti, strutturati e specializzandi che dedicano la propria vita alla crescita scientifica e sanitaria del nostro Paese. 


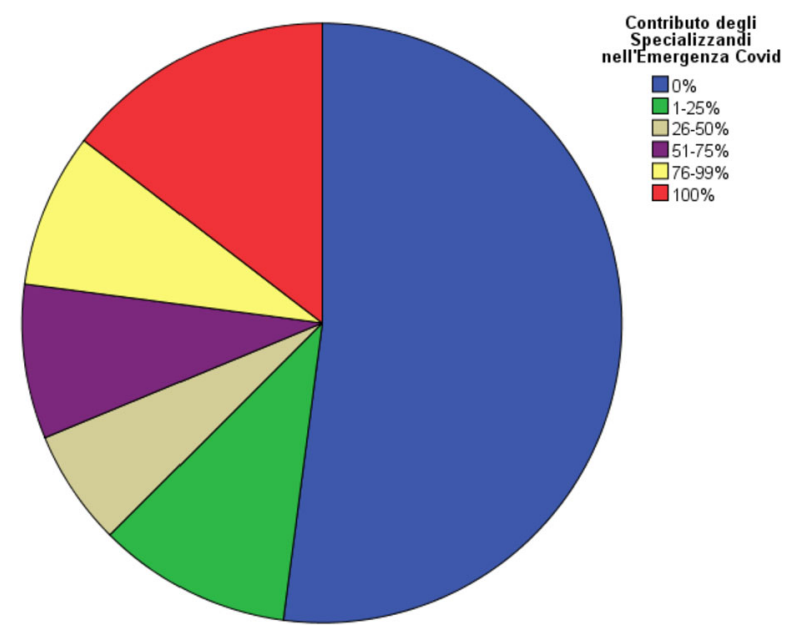

Fig. 5 Gestione ricoveri in DH endocrino-metabolico

Ringraziamenti Un ringraziamento particolare va alla Dottoressa Carlotta Pozza che ha generato il modulo web della survey e ha contribuito all' analisi dei dati.

Conflitto di interesse Gli autori Andrea M. Isidori, Emmanuele A. Jannini, Andrea Lenzi e Ezio Ghigo dichiarano di non avere conflitti di interesse.

Consenso informato Lo studio presentato in questo articolo non ha richiesto sperimentazione umana.
Studi sugli animali Gli autori di questo articolo non hanno eseguito studi sugli animali.

Nota della casa editrice Springer Nature rimane neutrale in riguardo alle rivendicazioni giurisdizionali nelle mappe pubblicate e nelle affiliazioni istituzionali.

\section{Bibliografia}

1. Fadini GP, Morieri ML, Longato E, Avogaro A (2020) Prevalence and impact of diabetes among people infected with SARS-CoV-2. J Endocrinol Invest. https://doi.org/10.1007/s40618-020-01236-2

2. Scaroni C, Armigliato M, Cannavo S (2020) COVID-19 outbreak and steroids administration: are patients treated for Sars-Cov-2 at risk of adrenal insufficiency? J Endocrinol Invest. https://doi.org/ 10.1007/s40618-020-01253-1

3. Trimarchi F (2020) An invisible hand has guided clinical endocrinology toward an unexpected future. J Endocrinol Invest. https:// doi.org/10.1007/s40618-020-01256-y

4. Isidori AM, Arnaldi G, Boscaro M et al (2020) COVID-19 infection and glucocorticoids: update from the Italian Society of Endocrinology Expert Opinion on steroid replacement in adrenal insufficiency. J Endocrinol Invest. https://doi.org/10.1007/ s40618-020-01266-w

5. Paoli D, Pallotti F, Colangelo S et al (2020) Study of SARS$\mathrm{CoV}-2$ in semen and urine samples of a volunteer with positive rino-pharyngeal swab. J Endocrinol Invest. https://doi.org/10.1007/ s40618-020-01261-1 